\title{
Propriedades físico-mecânicas da madeira de Eucalyptus alba para construção civil
}

\author{
Marta Cristina de Jesus Albuquerque Nogueira ${ }^{1}$, Victor Almeida De Araujo ${ }^{2 *}$, Juliano Souza \\ Vasconcelos ${ }^{3}$, Fabio Prataviera ${ }^{4}$, Julio Cezar Souza Vasconcelos ${ }^{4}$, José Nilton da Cruz ${ }^{5}$, André Luis \\ Christoforo ${ }^{6}$, Francisco Antonio Rocco Lahr ${ }^{7}$ \\ ${ }^{1}$ Departamento de Arquitetura, Universidade Federal de Mato Grosso, Cuiabá, Mato Grosso, Brasil. \\ ${ }^{2}$ Grupo de Pesquisa LIGNO, Itapeva, São Paulo, Brasil. \\ ${ }^{3}$ Faculdade de Ciências Agronômicas, Universidade Estadual Paulista (UNESP), Itapeva, São Paulo, Brasil. \\ ${ }^{4}$ Escola Superior de Agricultura Luiz de Queiroz (ESALQ), Universidade de São Paulo (USP), Piracicaba, São Paulo, Brasil. \\ ${ }^{5}$ Departamento de Estatística, Universidade Federal de Mato Grosso, Cuiabá, Mato Grosso, Brasil. \\ ${ }^{6}$ Departamento de Engenharia Civil, Universidade Federal de São Carlos (UFSCar), São Carlos, São Paulo, Brasil. \\ ${ }^{7}$ Departamento de Engenharia de Estruturas, Universidade de São Paulo (USP), São Carlos, São Paulo, Brasil.
}

\begin{abstract}
RESUMO As espécies de eucalipto têm se tornado uma matéria-prima multiuso. Algumas espécies possuem baixa abrangência no emprego como produtos duráveis. Uma variedade pouco utilizada é o Eucalyptus alba, apesar de sua popularidade nas empresas férreas. Este estudo objetivou investigar dezesseis propriedades físico-mecânicas do E. alba, visando ampliar o seu uso na construção. Os ensaios foram realizados para diferentes teores de umidade (12 e 30\%). Todos os resultados foram analisados pelo teste t. A densidade aparente, a compressão paralela no módulo de ruptura, a compressão paralela e a tração paralela no módulo de elasticidade, o fendilhamento, o cisalhamento, e as durezas normal e paralela revelaram um incremento com a redução de umidade. Os resultados obtidos nas propriedades de cisalhamento, fendilhamento e durezas normal e paralela possibilitam o uso da madeira de E. alba na construção civil para a produção de pisos estruturais.
\end{abstract}

Palavras-chave: Eucalipto branco; madeira serrada; piso de madeira; construção; dureza.

\section{Physical and mechanical properties of Eucalyptus alba wood for civil construction}

\begin{abstract}
Eucalypt species have become a multiple raw material. Some species have low coverage in the use as durable goods. Low-used variety is the Eucalyptus alba, despite its popularity in railway companies. This study aimed to investigate sixteen physical-mechanical properties of E. alba, aiming to enlarge its use in civil construction. Tests were carried out for different moisture contents (12 and 30\%). All results were analyzed by t-test. Apparent density, parallel compression in rupture moduli, parallel compression and tensile in elasticity moduli, cleavage, shear stress, and perpendicular and parallel hardness revealed an increasing with the moisture reduction. Obtained results in properties of shear stress, cleavage and parallel and perpendicular hardness enable E. alba wood utilization in construction for structural flooring production.
\end{abstract}

Keywords: White gum; lumber; wooden flooring; construction; hardness.

\section{Introdução}

A diversificação da utilização da madeira vem ganhando importância no cenário mundial por substituir produtos oriundos de fontes de recursos não renováveis como combustíveis e plásticos (LONGUE JUNIOR; COLODETTE, 2013). O surgimento do reflorestamento como forma de suprir as necessidades industriais proporcionou no uso de espécies de madeira não convencionais, isto é, exóticas 
(BOLGENHAGEN et al., 2015). No Brasil, esse processo silvicultural foi fortalecido para preservar diversas espécies nativas, em razão do emprego das espécies exóticas, especialmente Pinus spp. e Eucalyptus spp., para a indústria de base florestal-madeireira (DE ARAUJO et al., 2017).

O eucalipto foi introduzido no Brasil com um fim comercial em 1904, buscando suprir com matéria-prima as companhias férreas paulistas. Desde então, várias pesquisas têm demonstrado a versatilidade dessa madeira (WILCKEN et al., 2008). Porém, muitas variedades do eucalipto ainda demandam estudos para um emprego de maior valor agregado, e a espécie E. alba ainda é potencialmente pouco explorada.

O Eucalyptus alba Reinw. ex Blume é encontrado na Austrália (WILSON et al., 1990; TRAINOR, 2011), Indonésia, Timor-Leste (TRAINOR, 2011), Papua Nova Guiné (TRAINOR, 2011; NUBERG et al., 2015; NUBERG, 2015), Nigéria (OYEDEJI et al., 1999), Congo (DELWAULLE, 1985; LACLAU et al., 2000; CIMANGA et al., 2002; BOUILLET et al., 2002; LACLAU et al., 2005), Hong Kong (CORLETT, 1999), Argélia (BOUKHATEM et al., 2014), Angola, Costa Rica, Equador, Serra Leoa (FAO/UN, 1981), bem como nas várias pequenas ilhas asiáticas de Sunda (MOURA, 2004). Entretanto, é fortemente difundido no Brasil (BRASIL; FERREIRA, 1971; PÁSZTOR, 1975; FERREIRA, 1979; LACLAU et al., 2000), o qual se faz presente desde o início do Século XX (PÁSZTOR, 1975).

O Eucalyptus alba faz parte do grupo dos "Red Gums", que são espécies de madeira vermelha e casca dura, cor pardo avermelhado-clara e moderadamente dura (RIZZINI, 1971; GOLFARI et al., 1978). É empregado em óleos essenciais (OYEDEJI et al., 1999; CIMANGA et al., 2002; BOUKHATEM et al., 2014), medicinais (VALÉRIO et al., 2015) e taninos (NOMURA et al., 2005), bem como seu lenho é utilizado na produção de dormentes férreos (FERREIRA,
1979), energia (SANTOS; GOLDSTEIN JR, 2008; NUBERG et al., 2015; NUBERG, 2015), aglomerados e chapas (RIZZINI, 1971; GOLFARI et al., 1978).

A escassez de aplicações construtivas da madeira da espécie E. alba, tanto no Brasil como em outros países, reduz a sua popularização como matéria-prima industrial. Com isso, torna-se necessário o estudo do potencial dessa madeira, com o intuito de estimular o seu consumo em outros usos. Portanto, este estudo avaliou as propriedades físicomecânicas dessa respectiva madeira em diferentes teores de umidade, visando seu uso estrutural.

\section{Material e Métodos}

Este estudo contemplou a avaliação da madeira de Eucalyptus alba, convertida em amostras regularizadas e estabilizadas em dois teores de umidade (30\% saturado e $12 \%$ seco). Os testes mecânicos foram conduzidos em uma máquina universal de ensaios. No tocante as amostras estudadas, a Tabela 1 apresenta a origem das toras e seus aspectos.

O processo de caracterização físico-mecânica das madeiras oriundas dessas toras se estabeleceu similarmente àqueles adotados por Lahr et al. (2017) e Nogueira et al. (2018)

Tabela 1. Detalhes das amostras da madeira de Eucalyptus alba.

Table 1. Details of Eucalyptus alba wood samples.

\begin{tabular}{|c|c|c|c|c|}
\hline $\begin{array}{l}\text { Número } \\
\text { de } \\
\text { Árvores }\end{array}$ & $\begin{array}{c}\text { Quantidade } \\
\text { de } \\
\text { Vigas }\end{array}$ & Idade & $\begin{array}{c}\text { Diâmetro } \\
\text { (m) }\end{array}$ & $\begin{array}{c}\text { Região } \\
\text { de } \\
\text { Origem }\end{array}$ \\
\hline Tora 1 & 6 & 12 & 0,260 & $\begin{array}{c}\text { Guatapá, } \\
\text { Brasil }\end{array}$ \\
\hline Tora 2 & 4 & 12 & 0,240 & $\begin{array}{c}\text { Guatapá, } \\
\text { Brasil }\end{array}$ \\
\hline Tora 3 & 10 & 12 & 0,380 & $\begin{array}{c}\text { Guatapá, } \\
\text { Brasil }\end{array}$ \\
\hline Tora 4 & 6 & 12 & 0,255 & $\begin{array}{c}\text { Guatapá, } \\
\text { Brasil }\end{array}$ \\
\hline
\end{tabular}


Os ensaios físico-mecânicos para a caracterização estrutural dessa madeira foram prescritos pela norma brasileira ABNT NBR 7190 (1997): densidade básica $\left(\rho_{\mathrm{b}}\right)$ e densidade aparente $\left(\rho_{\text {ap }}\right)$ a $12 \%$ de umidade; resistências à compressão paralela às fibras $\left(\mathrm{f}_{\mathrm{c} 0}\right)$ e normal às fibras $\left(\mathrm{f}_{\mathrm{c} 90}\right)$; resistência ao cisalhamento paralelo às fibras $\left(f_{\mathrm{v} 0}\right)$; resistências à tração paralela $\left(f_{t 0}\right)$ e normal às fibras $\left(f_{t 90}\right)$; módulo de elasticidade na compressão paralela $\left(\mathrm{E}_{\mathrm{c} 0}\right)$ e normal $\left(\mathrm{E}_{\mathrm{c} 90}\right)$ às fibras; tenacidade $(\mathrm{W})$; fendilhamento $\left(\mathrm{f}_{\mathrm{CL}}\right)$; dureza $\left(\mathrm{f}_{\mathrm{H}}\right)$ paralela e perpendicular. As amostras foram confeccionadas seguindo os preceitos do anexo B da norma supracitada, cujas escolhas se basearam naquelas sem a presença de imperfeições como nós, falhas, medula, empenamentos e rachaduras. Assim, foram obtidos corpos de prova isentos de defeitos.

Com o fim de verificar a melhor condição estrutural da madeira de Eucalyptus alba, os ensaios foram realizados com as amostras de madeira em dois diferentes teores de umidade: o primeiro em um nível saturado e verde (30\%); e o segundo em um ponto padrão ao teor de umidade de equilíbrio (12\%) segundo a norma ABNT NBR 7190 (1997). Para estabelecer uma ampla amostragem, ao redor de 764 determinações foram realizadas com o E. alba. Para investigar a influência da umidade nessa madeira, $o$ teste $t$ foi aplicado com a admissão das seguintes condições: amostras serem independentes, aleatorização no processo de amostragem e sua normalidade. Duas hipóteses foram consideradas nessa análise: a não diferença das médias $\left(\mathrm{H}_{0}: \mu_{1}=\mu_{2}\right)$ e a diferença entre as médias $\left(\mathrm{H}_{1}: \mu_{1} \neq \mu_{2}\right)$. A decisão foi feita com base no P-valor associado ao teste, assumindo o nível de 5\% de probabilidade de erro. A rejeição da hipótese de igualdade de médias ocorre quando $\mathrm{H}_{0}$ foi de $5 \%$ ou menos (P-valor $\left.<0,005\right)$.

\section{Resultados e Discussão}

A Tabela 2 mostra um decréscimo do teor de umidade da madeira de Eucalyptus alba avaliada de 30\% (saturado) para o padrão da norma ABNT NBR 7190 (1997) de 12\% (teor de umidade de equilíbrio); a densidade aparente diminuiu $32,38 \%\left(0,34 \mathrm{~g} / \mathrm{cm}^{3}\right)$. Como a densidade básica é idêntica em qualquer teor de umidade, o estudo somente apontou seu valor na condição seca. Mediante o teste $t$, verificou-se que a densidade aparente sofreu influência em sua propriedade quando foi submetida à diminuição da umidade (de 30\% para $12 \%)$.

Em um comparativo com a literatura para ensaios similares, a redução da densidade aparente diante da redução da umidade também foi verificada por Nogueira et al. (2018) para a madeira de Eucalyptus umbra. Esses autores identificaram um decréscimo em menor escala. No caso da densidade básica para a condição seca a 12\% (Tabela 2), a mesma foi $20 \%$ menor que a espécie de E. umbra (NOGUEIRA et al., 2018) e 1,75\% menor que a madeira de $E$. urophylla (LAHR et al., 2017), ambas igualmente no mesmo parâmetro de umidade final.

Tabela 2. Resultados para a densidade da madeira de Eucalyptus alba.

Table 2. Results of Eucalyptus alba wood density.

\begin{tabular}{|c|c|c|c|c|c|}
\hline Característica & $\begin{array}{l}\text { TU } \\
(\%)\end{array}$ & $\mathbf{n}$ & $\mathbf{M}$ & $d p$ & $\begin{array}{l}\text { Valor } \\
\text { de p }\end{array}$ \\
\hline \multirow{2}{*}{$\begin{array}{l}\text { Densidade Aparente } \\
\left(\mathrm{g} / \mathrm{cm}^{3}\right)\end{array}$} & 30 & 25 & 1,05 & 0,05 & \multirow{2}{*}{0,0000} \\
\hline & 12 & 25 & 0,71 & 0,09 & \\
\hline $\begin{array}{c}\text { Densidade Básica } \\
\left(\mathrm{g} / \mathrm{cm}^{3}\right)\end{array}$ & 12 & 25 & 0,56 & 0,07 & ----- \\
\hline
\end{tabular}

TU: teor de umidade; $\mathrm{n}$ : repetições; $\mathrm{M}$ : médias das propriedades; dp: desvio padrão.

A Tabela 3 indica acréscimos para todas as propriedades de resistência no módulo de ruptura: 29,18\% (13,8 MPa) na compressão paralela, $7,14 \%(0,3 \mathrm{MPa})$ na compressão normal, 9,03\% (6,3 $\mathrm{MPa})$ na tração paralela, 30,77\% (1,2 
$\mathrm{MPa})$ na tração normal e 6,1\% (8,24 MPa) na flexão estática.

Diante disso, verificou-se, pelo teste $t$, que a compressão paralela consistiu na única propriedade que sofreu influência com a redução de umidade de 30 para 12\%, enquanto que algo similar não ocorreu com os módulos de ruptura na compressão normal, tração paralela, tração normal e flexão estática. Para uma condição similar da redução do teor de umidade, o aumento dos módulos de ruptura representou uma situação igualmente determinada por Lahr et al. (2017) para o E. urophylla.

A Tabela 4 retrata que os módulos de elasticidade apresentaram aumentos em suas propriedades, na ordem de $30,87 \%$ (4139,1 MPa) na compressão paralela, 12,21\% (2061,3
$\mathrm{MPa})$ na tração paralela, $11,97 \%(1581,1 \mathrm{MPa})$ na flexão estática. Somente a compressão normal apresentou uma diminuição de 6,49\% (25,6 MPa). Com o teste t, foi possível avaliar que a compressão paralela e a tração paralela sofreram influência em suas propriedades com a redução do teor de umidade observado (de 30 para 12\%). Porém, os módulos de elasticidade na compressão normal e na flexão estática não rejeitaram a hipótese nula de igualdade das médias. Similar à observação constatada por Lahr et al. (2017) em seu estudo para a espécie E. urophylla, a maior parte dos módulos de elasticidade obtidos para o eucalipto aqui avaliado também indicaram incrementos com a redução da umidade de 30 para $12 \%$.

Tabela 3. Resultados para os módulos de ruptura do Eucalyptus alba.

Table 3. Results of Eucalyptus alba wood moduli of rupture.

\begin{tabular}{|c|c|c|c|c|c|}
\hline Característica & TU (\%) & $\mathbf{n}$ & $\mathbf{M}$ & dp & P-valor \\
\hline \multirow{2}{*}{ Compressão Paralela (MPa) } & 30 & 25 & 33,5 & 17,6 & \multirow{2}{*}{0,0010} \\
\hline & 12 & 24 & 47,3 & 7,4 & \\
\hline \multirow{2}{*}{ Compressão Normal (MPa) } & 30 & 25 & 4,2 & 1,3 & \multirow{2}{*}{0,4101} \\
\hline & 12 & 23 & 3,9 & 1,2 & \\
\hline \multirow{2}{*}{ Tensão Paralela (MPa) } & 30 & 23 & 63,5 & 25,8 & \multirow{2}{*}{0,4273} \\
\hline & 12 & 24 & 69,8 & 28,1 & \\
\hline \multirow{2}{*}{ Tensão Normal (MPa) } & 30 & 23 & 2,7 & 1,0 & \multirow{2}{*}{0,0494} \\
\hline & 12 & 26 & 3,9 & 2,8 & \\
\hline \multirow{2}{*}{ Flexão Estática (MPa) } & 30 & 21 & 67,9 & 13,4 & \multirow{2}{*}{0,2647} \\
\hline & 12 & 22 & 74,0 & 21,2 & \\
\hline
\end{tabular}

TU: teor de umidade; n: repetições; M: médias das propriedades; dp: desvio padrão.

Tabela 4. Resultados para os módulos de elasticidade do Eucalyptus alba.

Table 4. Results of Eucalyptus alba wood moduli of elasticity.

\begin{tabular}{|c|c|c|c|c|c|}
\hline Característica & TU (\%) & $\mathbf{n}$ & $\mathbf{M}$ & dp & P-valor \\
\hline \multirow{2}{*}{ Compressão Paralela (MPa) } & 30 & 25 & 9270,3 & 3230,5 & \multirow{2}{*}{0,0000} \\
\hline & 12 & 24 & 13409,4 & 2423,0 & \\
\hline \multirow{2}{*}{ Compressão Normal (MPa) } & 30 & 25 & 420,3 & 128,7 & \multirow{2}{*}{0,4820} \\
\hline & 12 & 23 & 394,7 & 121,5 & \\
\hline \multirow{2}{*}{ Tração Paralela (MPa) } & 30 & 23 & 14825,3 & 3248,7 & \multirow{2}{*}{0,0304} \\
\hline & 12 & 24 & 16886,6 & 3064,7 & \\
\hline \multirow{2}{*}{ Flexão Estática (MPa) } & 30 & 21 & 11625,5 & 1967,3 & \multirow{2}{*}{0,0373} \\
\hline & 12 & 22 & 13206,6 & 2783,8 & \\
\hline
\end{tabular}

TU: teor de umidade; n: repetições; M: médias das propriedades; dp: desvio padrão.

A Tabela 5 indica aumentos nas propriedades de cisalhamento $(38,36 \%$ ou $6,1 \mathrm{MPa})$, fendilhamento $(31,63 \%$ ou $0,31 \mathrm{MPa})$, dureza normal $(38,40 \%$ ou $2,78 \mathrm{kN})$ e dureza paralela $(33,56 \%$ ou $1,97 \mathrm{kN})$. Somente o módulo de tenacidade diminuiu, nesse caso, na ordem de $15,29 \%(1,3$ 
N.m). A análise do teste $t$ indicou que as propriedades de cisalhamento, fendilhamento, dureza normal e dureza paralela sofreram influência com a redução do teor de umidade estudado. Por outro lado, a tenacidade não indicou alterações na condição estudada. Em confronto ao estudo de Lahr et al. (2017) para o mesmo propósito da investigação de suas propriedades após a redução de umidade de 30 para 12\%, a madeira de E. alba igualmente indicou melhorias, tal como a variedade E. urophylla em análise pelos autores supracitados, em suas propriedades de cisalhamento, fendilhamento e durezas normal e paralela.

De modo geral, com o suporte da análise estatística do teste $t$, das quatorze propriedades mecânicas estudadas para a madeira de Eucalyptus alba, sete delas indicaram mudanças significativas com a diminuição do teor de umidade, as quais tiveram um incremento em suas resistências. A compressão paralela no módulo de ruptura, a compressão paralela e a tração paralela no módulo de elasticidade, o cisalhamento, o fendilhamento e as durezas normal e paralela revelaram um aumento de suas propriedades na condição de redução do teor de umidade de 30 para 12\%. Excluindo as compressões normais para os módulos de ruptura e elasticidade, as quais indicaram reduções muito pequenas, todas as outras doze propriedades mecânicas partilharam melhorias perceptíveis, seguindo os preceitos da norma ABNT NBR 7190 (1997) para a condição avaliada, permitindo o emprego desse eucalipto estudado em elementos para em estruturas de madeira. Pela Tabela 3, observa-se que os resultados obtidos para a resistência à compressão paralela às fibras permitem considerar o E. alba como enquadrado na "Classe C40", isto é, a segunda classe mais elevada estabelecida pela norma ABNT NBR 7190 (1997). Esses resultados permitem registrar que o Eucalyptus alba apresentou resultados equivalentes a outras espécies de madeira usualmente empregadas na construção de estruturas no Brasil, como o angelim araroba (Vataireopsis araroba), branquilho (Terminalia sp.) e cupiúba (Goupia glabra), com base no que consta no "Anexo E” dessa mesma norma brasileira.

Em suma, os resultados obtidos (Tabelas 3 a 5) ainda revelaram que a madeira de Eucalyptus alba possui um comportamento mecânico equivalente ao reportado por Lahr et al. (2017) para a variedade de Eucalyptus urophylla, alcançando valores bastante similares para as seguintes propriedades: compressão paralela, tensão normal e flexão estática nos módulos de ruptura; compressão paralela, tensão paralela e flexão estática nos módulos de elasticidade; cisalhamento, fendilhamento e durezas normal e paralela.

Tabela 5. Resultados para outras propriedades de resistência do Eucalyptus alba.

Table 5. Results of other strength properties of Eucalyptus alba wood.

\begin{tabular}{|c|c|c|c|c|c|}
\hline Característica & TU (\%) & $\mathbf{n}$ & MO & dp & Valor de $p$ \\
\hline \multirow{2}{*}{ Cisalhamento (MPa) } & 30 & 25 & 9,8 & 1,7 & \multirow{2}{*}{0,0000} \\
\hline & 12 & 24 & 15,9 & 1,6 & \\
\hline \multirow{2}{*}{ Fendilhamento (MPa) } & 30 & 25 & 0,67 & 0,2 & \multirow{2}{*}{0,0000} \\
\hline & 12 & 22 & 0,98 & 0,21 & \\
\hline \multirow{2}{*}{ Dureza Normal (kN) } & 30 & 25 & 4,46 & 0,62 & \multirow{2}{*}{0,0000} \\
\hline & 12 & 24 & 7,24 & 1,05 & \\
\hline \multirow{2}{*}{ Dureza Paralela $(\mathrm{kN})$} & 30 & 25 & 3,90 & 0,70 & \multirow{2}{*}{0,0000} \\
\hline & 12 & 24 & 5,87 & 1,75 & \\
\hline \multirow{2}{*}{ Tenacidade (N.m) } & 30 & 23 & 8,5 & 5,1 & \multirow{2}{*}{0,3645} \\
\hline & 12 & 24 & 7,2 & 4,6 & \\
\hline
\end{tabular}

TU: teor de umidade; n: repetições; $\mathrm{M}_{\mathrm{O}}$ : médias das propriedades; dp: desvio padrão 
Diante disso, por meio dos resultados obtidos, as seguintes considerações finais são indicadas:

Levando em consideração que o objetivo do estudo foi avaliar as propriedades físico-mecânicas do E. alba, em dois níveis de umidade, buscando confirmar a possibilidade de seu emprego estrutural, os resultados permitem admitir a adequação da espécie para tal finalidade;

Levando em consideração a lacuna existente na literatura brasileira em relação ao comportamento físico-mecânico da espécie estudada, a opção, nessa fase da pesquisa, foi apresentar as propriedades físico-mecânicas que corroborassem com a hipótese confirmada no item anterior, o que poderá viabilizar sua maior utilização estrutural;

A relevância de abordagem a respeito das peculiaridades químicas e anatômicas da espécie seria expressiva se não houvesse sido detectada, na análise estatística, diferença significativa entre as propriedades estudadas, quando considerados os dois níveis de umidade adotados.

\section{Conclusões}

Diante do exposto, pode-se verificar que:

Diante da atual busca por espécies exóticas não tradicionais, a madeira de Eucalyptus alba pode ser eficientemente utilizada na construção civil brasileira, ao passo que as suas propriedades mecânicas atenderam as prescrições da norma brasileira voltada para as estruturas de madeira, bem como indicaram aumentos perceptíveis das mesmas durante a perda de umidade observada;

Os resultados obtidos nas propriedades de resistência ao cisalhamento, fendilhamento e às durezas normal e paralela possibilitam, inclusive, o uso eficiente dessa espécie em aplicações estruturais, especialmente, na produção de pisos, bases portantes para pisos, decks e assoalhos, visto que atendem as prescrições da norma brasileira.

\section{Referências}

ASSOCIAÇÃO BRASILEIRA DE NORMAS TÉCNICAS. NBR 7190: Projeto de estruturas de madeira. Rio de Janeiro, 1997.

BOLGENHAGEN, A.; HORST, D. J.; PETTER, R. R. H; BORMANN, O. R. Assessing the physical and mechanical properties of three native species from Southern Brazil. Ciência da Madeira, v. 6, n. 1, p. 23-30, 2015.

BOUILLET, J. P.; LACLAU, J. P.; ARNAUD, M.; M'BOU, A. T.; SAINT-ANDRÉ, L.; JOURDAN, C. Changes with age in the spatial distribution of roots of Eucalyptus clone in Congo: Impact on water and nutrient uptake. Forest Ecology and Management, v. 171, n. 1-2, p. 43-57, 2002.

BOUKHATEM, M. N.; AMINE, F. M.; KAMELI, A.; SAIDI, F.; WALID, K.; MOHAMED, S. B. Quality assessment of the essential oil from Eucalyptus globulus Labill of Blida (Algeria) origin. International Letters of Chemistry, Physics and Astronomy, v. 17, n. 3, p. 303-315, 2014.

BRASIL, M. A. M.; FERREIRA, M. Variação da densidade básica da madeira de Eucalyptus alba Reinw, Eucalyptus saligna Smith e Eucalyptus grandis aos 5 anos de idade em função do local e do espaçamento. IPEF, n. 2-3, p. 129-149, 1971.

CIMANGA, K.; KAMBU, K.; TONA, L.; APERS, S.; DE BRUYNE, T.; HERMANS, N.; TOTTÉ, J.; PIETERS, L.; VLIETINCK, A. J. Correlation between chemical composition and antibacterial activity of essential oils of some aromatic medicinal plants growing in the Democratic Republic of Congo. Journal of Ethnopharmacology, v. 79, n. 2, p. 213-220, 2002.

CORLETT, R. T. Environmental forestry in Hong Kong: 1871-1997. Forest Ecology and Management, v. 116, p. 93$105,1999$.

DE ARAUJO, V. A.; GARCIA, J. N.; CORTEZ-BARBOSA, J.; GAVA, M.; SAVI, A. F.; MORALES, E. A. M.; LAHR, F. A. R.; VASCONCELOS, J. S.; CHRISTOFORO, A. L. Importância da madeira de florestas plantadas para a indústria de manufaturados. Pesquisa Florestal Brasileira, v. 37, n. 90, p. 157-168, 2017.

DELWAULLE, J. C. Plantations clonales d'Eucalyptus hybrides au Congo. Revue Bois et Forêts des Tropiques, n. 208, p. 37-42, 1985. 
FOOD AND AGRICULTURE ORGANIZATION OF THE UNITED NATIONS (FAO/UN). Eucalypts for planting. 2. ed. Roma, Itália: FAO/UN, 1981. 677 p.

FERREIRA, M. Escolha de espécies de eucalipto. Circular técnica, n. 47. Piracicaba: IPEF, 1979. 17 p.

GOLFARI, L.; CASER, R. L.; MOURA, V. P. G. Zoneamento ecológico esquemático para reflorestamento no Brasil (2aaproximaçao). Série Técnica, n. 11. Belo Horizonte: PRODEPEF-Centro de Pesquisa Florestal do Cerrado, 1978. $66 \mathrm{p}$.

LACLAU, J. P.; BOUILLET, J. P.; RANGER, J. Dynamics of biomass and nutrient accumulation in a clonal plantation of Eucalyptus in Congo. Forest Ecology and Management, v. 128, p. 181-196, 2000.

LACLAU, J. P.; RANGER, J.; DELEPORTE, P.; NOUVELLON, Y.; SAINT-ANDRÉ, L.; MARLET, S.; BOUILLET, J. P. Nutrient cycling in a clonal stand of Eucalyptus and an adjacent savanna ecosystem in Congo: 3. Input-output budgets and consequences for the sustainability of the plantations. Forest Ecology and Management, v. 210, n. 1-3, p. 375-391, 2005.

LAHR, F. A. R.; NOGUEIRA, M. C. J. A.; DE ARAUJO, V. A.; VASCONCELOS, J. S.; CHRISTOFORO, A. L. Physicalmechanical characterization of Eucalyptus urophylla wood. Engenharia Agrícola, v. 37, n. 5, p. 900-906, 2017.

LONGUE JUNIOR, D.; COLODETTE, J. L. Importância e versatilidade da madeira de eucalipto para a indústria de base florestal. Pesquisa Florestal Brasileira, v. 33, n. 76, p. 429438, 2013.

MOURA, V. P. G. O germoplasma de Eucalyptus urophylla

S. T. Blake no Brasil. Comunicado Técnico, n. 111. Brasília: EMBRAPA, 2004. 12 p.

NOGUEIRA, M. C. J. A.; ALMEIDA, D. H.; VASCONCELOS, J. S.; ALMEIDA, T. H.; ARAUJO, V. A.; CHRISTOFORO, A. L.; LAHR, F. A. R. Properties of Eucalyptus umbra wood for timber structures. International Journal of Materials Engineering, v. 8, n. 1, p. 12-15, 2018.

NOMURA, N.; TSUKADA, H.; ICHIMATSU, D.; ITO, H.; YOSHIDA, T.; MIYAMOTO, K. I. Inhibition of epidermal growth factor-induced cell transformation by tannins. Phytochemistry, v. 66, n. 17, p. 2038-2046, 2005.
NUBERG, I. K. Developing the fuelwood economy of Papua New Guinea. Energy for Sustainable Development, v. 24, p. 9-18, 2015.

NUBERG, I. K.; GUNN, B.; TAVUNE, M.; SUMAREKE, A.; KRAVCHUK, O. Evaluation of short-rotation coppicing fuelwood production systems for Papua New Guinea. Biomass and Bioenergy, v. 78, p. 126-139, 2015.

OYEDEJI, A. O.; EKUNDAYO, O.; OLAWORE, O. N.; ADENIYI, B. A.; KOENIG, W. A. Antimicrobial activity of the essential oils of five Eucalyptus species growing in Nigeria. Fitoterapia, v. 70, p. 526-528, 1999.

PÁSZTOR, Y. P. C. Estudo comparativo do comportamento das espécies Eucalyptus alba Reinw e E. decaiseana Blune, na região de São Paulo. IPEF, n. 11, p. 1-16, 1975.

RIZZINI, C. T. Arvores e madeiras úteis do Brasil; manual de dendrología brasileira. São Paulo, Editora Blucher, 1971. 294 p.

SANTOS, F. J.; GOLDSTEIN JR, L. Experimental aspects of biomass fuels in a bubbling fluidized bed combustor. Chemical Engineering and Processing: Process Intensification, v. 47, n. 9-10, p. 1541-1549, 2008.

TRAINOR, C. R. New island records of Eucalyptus alba sensu lato for Damar and Romang, Lesser Sundas, Indonesia. Northern Territory Naturalist, v. 23, p. 45-53, 2011.

VALÉRIO, E. S.; BARBOSA, W. L. R.; VASCONCELOS, F.; FINGER, R. M.; MUZITANO, M. F.; HEGGDORNE, M.; PIVA, L. Avaliação da atividade do extrato hidroetanólico de Chenopodium ambrosioides L. e de Eucalyptus alba Reinw ex Blume, frente a cepas de Mycobacterium sp in vitro. Revista de Biotecnologia \& Ciência, v. 4, n. 1, p. 1-1, 2015.

WILCKEN, C. F.; LIMA, A. C. V.; DIAS, T. K. R.; MASSON, M. V.; FERREIRA FILHO, P. J. F.; POGETTO, M. H. F. A. D. Guia prático de manejo de plantações de eucalipto. Botucatu: FEPAF/UNESP, 2008. 25 p.

WILSON, B. A.; BROCKLEHURST, P. S.; CLARK, M. J.; DICKINSON, K. J. M. Vegetation Survey of the Northern Territory, Australia. Palmerston, Australia: Conservation Commission of the Northern Territory, 1990. 222 p. 